(2)

CASE REPORT

\title{
Intermittent acute angle closure glaucoma and chronic angle closure following topiramate use with plateau iris configuration
}

This article was published in the following Dove Press journal:

Clinical Ophthalmology

17 July 2014

Number of times this article has been viewed

\section{Lamise Z Rajjoub \\ Nisha Chadha \\ David A Belyea}

Department of Ophthalmology, The George Washington University, Washington, DC, USA
Correspondence: David A Belyea Department of Ophthalmology,

The George Washington University, 2I50 Pennsylvania Ave NW, Suite 2A, Washington, DC 20037, USA

Tel + I $20274 \mid 2800$

Fax + $20274|282|$

Email dbelyea@mfa.gwu.edu

\begin{abstract}
This is a case report describing recurrent intermittent acute angle closure episodes in the setting of topiramate use in a female suffering from migraines. Despite laser peripheral iridotomy placement for the pupillary block component, and the discontinuation of topiramate, the acute angle closure did not resolve in the left eye with chronic angle closure and the patient required urgent trabeculectomy. The right eye responded to laser peripheral iridotomy immediately and further improved after the cessation of topiramate. While secondary angle closure glaucoma due to topiramate use has been widely reported, its effects in patients with underlying primary angle closure glaucoma have not been discussed. Our report highlights the importance of recognizing the often multifactorial etiology of angle closure glaucoma to help guide clinical management.
\end{abstract}

Keywords: angle closure glaucoma, plateau iris, topiramate, secondary glaucoma, drug-induced glaucoma

\section{Introduction}

Topiramate is a sulfamate-substituted monosaccharide, used in the treatment of epilepsy, depression, migraine, and neuropathic pain. Secondary angle closure glaucoma due to topiramate is well recognized and has been related to supraciliary effusions and forward rotation of ciliary processes. Furthermore, the medication is associated with drug-induced myopia, due to anterior displacement of the iris-lens diaphragm. In most cases, this secondary angle closure glaucoma and myopic shift resolves rapidly with discontinuation of the medication and management of intraocular pressure (IOP) with topical therapy.

\section{Case report}

A 36-year-old Caucasian female presented to the emergency department with a oneday history of severe headache, abdominal pain, and decreased vision in the left eye (OS). The patient reported that she had been suffering from headaches for the past 11 months, for which she was being treated by a neurologist. During previous headache episodes, she experienced decreased vision in either eye and a frontal headache centered around or behind the affected eye lasting 8-10 hours. Her medical history was significant for Crohn's disease, migraines, erosive gastritis, and kidney stones. Her personal and ocular history was unremarkable, and she had never worn glasses. Her medications included mesalamine, sumatriptan, topiramate (prescribed 10 months prior by neurologist), dexlansoprazole, oxycodone/acetaminophen, ondansetron, and promethazine. 
On examination, visual acuity was 20/30 in the right eye (OD) and count fingers at 6 inches OS. IOP via TONO-PEN (TONO-PEN AVIA Tonometer, Reichert Technologies, Depew, NY, USA) was $31 \mathrm{mmHg}$ OD, and $53 \mathrm{mmHg}$ OS. The left pupil was mid-dilated with a reverse afferent pupillary defect. Anterior segment examination OD was significant for a narrow anterior chamber. Anterior segment examination OS revealed mild conjunctival injection, diffuse microcystic edema of the cornea, and a narrow anterior chamber with 1-2+ pigmented cells. Gonioscopy revealed appositionally closed angles bilaterally, which did not open with goniocompression OS, and opened with a plateau iris configuration (double hump sign) OD. Undilated funduscopic examination findings were asymmetric with a cup-disc ratio of $0.3 \mathrm{OD}$, and $0.7 \mathrm{OS}$.

Bilateral secondary angle closure with acute angle closure glaucoma OS was diagnosed. Topical therapy was initiated with brimonidine/timolol and dorzolamide ophthalmic drops. While IOP OD improved to $18 \mathrm{mmHg}$, the pressure remained elevated OS at $50 \mathrm{mmHg}$. The patient had a history of kidney stones, therefore oral and intravenous carbonic anhydrase inhibitors were avoided. The patient was also advised to discontinue use of topiramate. While the mechanism of acute angle closure was thought to be secondary to topiramate use, underlying chronic angle closure could not be ruled out given the history of repeated episodes of vision loss during headaches, even prior to initiation of topiramate and the advanced cupping of the optic nerve OS. Ultrasound biomicroscopy was not available, therefore, based on the clinical examination (gonioscopy), plateau iris angle closure was suspected while recognizing that secondary angle closure from ciliary body effusion may also be a factor. Therefore, in addition to advising discontinuation of topiramate, a laser peripheral iridotomy (PI) was performed OS with reduction in IOP to $22 \mathrm{mmHg}$ OS. A prophylactic PI was also placed OD with posttreatment IOP of $12 \mathrm{mmHg}$ OD.

Twelve hours later, visual acuity was 20/30 OD, and 20/70 OS with IOP of $09 \mathrm{mmHg}$ OD, and $34 \mathrm{mmHg}$ OS, despite the discontinuation of topiramate and the use of topical bimatoprost and brimonidine/timolol. On anterior segment examination OS, the anterior chamber appeared deeper centrally. Gonioscopy revealed plateau iris configuration with patent iridotomies and an open angle OD, but peripheral anterior synechiae with a closed angle despite goniocompression OS. At this time, topical dorzolamide was added to treat the high IOP OS.
Because the patient was visiting from out of state and had to return home immediately, follow-up with a glaucoma specialist was arranged. At the patient's initial consultation with the glaucoma specialist 2 days later, visual acuity was 20/20 OD, and 20/40 OS with an IOP of $12 \mathrm{mmHg}$ OD, and $50 \mathrm{mmHg}$ OS. An anterior chamber paracentesis was done OS and the IOP normalized. The plan was to proceed with a trabeculectomy the following day, however, this was postponed as the patient was admitted to the hospital for a flare-up of her Crohn's disease. Throughout her hospitalization, IOP remained well controlled in the range of 10-12 mmHg OU without topical medications OD, and on topical bimatoprost, brimonidine/timolol and dorzolamide OS. One day after the patient was discharged from the hospital, she was found to have elevated IOP $53 \mathrm{mmHg}$ OS with a patent iridotomy. A second anterior chamber paracentesis was done and again the IOP had been normalized. The following day, the IOP was elevated again to $51 \mathrm{mmHg}$ OS and the patient underwent an urgent trabeculectomy with mitomycin C OS. Postoperatively, the patient's visual acuity OS returned to 20/20 and IOP OS was controlled with trabeculectomy in the range of $09-10 \mathrm{mmHg}$.

\section{Discussion}

Banta et $\mathrm{al}^{1}$, Sanka et $\mathrm{al}^{2}$, and Rhee et $\mathrm{al}^{3}$ were among the first to report cases of secondary angle closure glaucoma associated with the use of topiramate in 2001. They described uveal effusions, ciliary process swelling, and forward displacement of the iris-lens diaphragm leading to myopic shift and secondary angle closure glaucoma. By 2003, Fraunfelder et $\mathrm{al}^{4}$ reviewed 115 case reports of topiramate induced angle closure glaucoma. They found that $85 \%$ of cases of topiramate associated secondary angle closure glaucoma occurred within the first 2 weeks of treatment. There were 17 reports of acute bilateral myopia (up to 8.75 diopters), and vision returned to normal within a few days to a few weeks of discontinuation of topiramate. Nine cases had documented suprachoroidal effusions. Management of topiramate induced secondary angle closure glaucoma based on Fraunfelder et $\mathrm{al}^{4}$ included: 1) prompt discontinuation of topiramate, as IOP decreases rapidly after the medication is stopped; 2) maximal medical therapy (oral medications and aqueous suppressants), and avoiding topical miotics in order to avoid relative pupillary block. Furthermore, laser PI was not found to be beneficial if glaucoma was only associated with topiramate. Topiramate induced secondary angle closure glaucoma has been found to resolve within 
24-48 hours of discontinuation of the mediation, while the myopia resolves within 1-2 weeks. ${ }^{5}$

We present the case of a 36-year-old Caucasian female with plateau iris configuration, acute onset angle closure, and acute angle closure superimposed on chronic angle closure in the setting of topiramate use. While it is difficult to prove the exact etiology of her angle closure without ultrasound biomicroscopy, which we did not have the luxury of obtaining during this patient's acute attack, it is presumed that her topiramate use may have contributed to the acute attack. Her acute change in vision in a previous emmetropic eye and improvement in visual acuity with discontinuation of the medication support this assumption. Regardless of the mechanism, this case raises a few notable points: 1) the multifactorial etiology of some forms of angle closure; 2) the prescription of topiramate in eyes with underlying narrow angles; and 3) the possibility of delayed secondary angle closure with topiramate use.

Our patient did not have a prior ophthalmology examination diagnosis of narrow angles or angle closure glaucoma, but had clinical symptoms of angle closure which preceded the topiramate use, and clinical findings including narrow angles (plateau iris OD) and chronic angle closure OS, which suggests multiple etiologies as an underlying mechanism for angle closure. Follow-up examination OD revealed a more open, narrow, non-occludable angle with a patent PI off topiramate but with a plateau iris configuration, which could explain the presenting angle closure OD, while the 360 degree peripheral anterior synechiae OS (likely from chronic intermittent angle closure episodes) explains the persistent elevated IOP and permanently closed angle OS. Finally, topiramate use could have further contributed to the angle closure precipitating the attack by a ciliary body effusion that led the patient to present to our emergency department. Of note, this patient was on other systemic medications with anticholinergic properties which could further contribute to her risk of angle closure. Her case highlights the often multifactorial nature of angle closure glaucoma that can indicate multiple therapeutic interventions. In this patient's case, while topiramate induced secondary angle closure was one suspected mechanism, a peripheral iridotomy was also warranted based on other clinical findings (plateau iris) and to help rule out aqueous misdirection once the topiramate was discontinued. Furthermore, when plateau iris is identified on gonioscopy but the patient is asymptomatic with a normal IOP, we typically attempt laser PI first. We find that this will often open up the angle and resolve the occludability. If, however, the peripheral iridotomy fails to open up the angle or the patient is symptomatic or develops elevated IOP, we would then perform peripheral iridoplasty. The multifactorial nature of this patient's angle closure also raises the question of whether this patient was a suitable candidate for topiramate therapy and whether her underlying narrow occludable angles should have been a relative contraindication to this drug. It suggests that perhaps, baseline ophthalmic examination with gonioscopy should be performed on all individuals being considered for topiramate therapy.

Finally, our patient had been receiving topiramate therapy for roughly 10 months, far longer than the average timeframe of 2 weeks post-initiation of therapy to induce secondary angle closure glaucoma, and by taking into account her history we suspect the patient was having angle closure symptoms prior to commencing topiramate. Only one such report exists by $\mathrm{Czyz}$ et $\mathrm{al}^{7}$ who reported a delayed onset of topiramate induced angle closure glaucoma, 262 days after initiation of therapy, which resolved with topical aqueous suppressants and discontinuation of topiramate. This finding suggests that patients on topiramate should be monitored and counseled regarding angle closure beyond the initial 2-4 weeks after initiation of therapy.

While angle closure secondary to topiramate therapy has been widely recognized as a potential side effect of this medication, our report suggests that perhaps baseline ophthalmic examination should be performed prior to initiation of therapy, and that findings of narrow occludable angles should be a relative contraindication to treatment with this medication. Patients with angle closure symptoms could be mistaken by non-ophthalmologists as suffering from migraines. Further analysis of combined-mechanism angle closure is warranted to elucidate whether exclusion of patients with narrow angle glaucoma from topiramate treatment would reduce the occurrence of this side effect.

\section{Disclosure}

None of the authors have grants or financial interests to report. This manuscript has not been previously presented. The authors have no other conflicts of interest to declare.

\section{References}

1. Banta JT, Hoffman K, Budenz DL, Ceballos E, Greenfield DS. Presumed topiramate-induced bilateral acute angle-closure glaucoma. Am J Ophthalmol. 2001;132(1):112-114.

2. Sankar PS, Pasquale LR, Grosskreutz CL. Uveal effusion and secondary angle-closure glaucoma associated with topiramate use. Arch Ophthalmol. 2001;119(8):1210-1211.

3. Rhee DJ, Goldberg MJ, Parrish RK. Bilateral angle-closure glaucoma and ciliary body swelling from topiramate. Arch Ophthalmol. 2001;119(11): 1721-1723. 
4. Fraunfelder FW, Fraunfelder FT, Keates EU. Topiramate-associated acute, bilateral, secondary angle-closure glaucoma. Ophthalmology. 2004; 111(1):109-111.

5. Medeiros FA, Zhang XY, Bernd AS, Weinreb RN. Angle-closure glaucoma associated with ciliary body detachment in patients using topiramate. Arch Ophthalmol. 2003;121(2):282-285.
6. Craig JE, Ong TJ, Louis DL, Wells JM. Mechanism of topiramateinduced acute-onset myopia and angle closure glaucoma. Am J Ophthalmol. 2004;137(1):193-195.

7. Czyz CN, Clark CM, Justice JD, Pokabla MJ, Weber PA. Delayed Topiramate-induced Bilateral Angle-Closure Glaucoma. J Glaucoma. Epub 2013 Apr 29.

\section{Publish your work in this journal}

Clinical Ophthalmology is an international, peer-reviewed journal covering all subspecialties within ophthalmology. Key topics include: Optometry; Visual science; Pharmacology and drug therapy in eye diseases; Basic Sciences; Primary and Secondary eye care; Patient Safety and Quality of Care Improvements. This journal is indexed on

\section{Dovepress}

PubMed Central and CAS, and is the official journal of The Society of Clinical Ophthalmology (SCO). The manuscript management system is completely online and includes a very quick and fair peer-review system, which is all easy to use. Visit http://www.dovepress.com/ testimonials.php to read real quotes from published authors. 